\title{
Prevalencia de comorbilidad depresiva en la enfermedad de Ménière
}

\section{Prevalence of comorbid depression in Ménière's disease}

\author{
Romina Ovalle A. ${ }^{1}$, Margarita Machuca B. ${ }^{2}$, Pablo Moreira Z. ${ }^{3}$, \\ Tamara Barría E. ${ }^{1}$, María Durán R. ${ }^{4}$, Jaime Osorio M. ${ }^{1,5}$
}

\section{Resumen}

Introducción: Existe relación entre la enfermedad de Ménière (EM) y los trastornos psiquiátricos. Objetivo: Evaluar si los pacientes con EM presentan mayor prevalencia de comorbilidad depresiva en comparación a lo descrito para la población general nacional. Material y Método: Estudio analítico transversal entre los años 2009-2016, en pacientes del Servicio de Otorrinolaringología del Hospital Barros Luco Trudeau con diagnóstico de EM definitivo. Se aplicó la escala del nivel funcional para EM, tinnitus handicap inventory, test de tamizaje de depresión de Goldberg y una evaluación por psiquiatra. Resultados: Se evaluaron 64 pacientes. Promedio de edad fue 52,5 años. La prevalencia de trastorno depresivo fue $71,8 \%$. De éstos, el 71,7\% fue diagnosticado antes del estudio y el 28,3\% restante durante el estudio. Conclusión: La prevalencia de comorbilidad depresiva en la EM es mayor que la descrita para la población general nacional. Determinar si la EM es la causante de esta prevalencia, o cada uno de sus síntomas por separado, requerirá de nuevos estudios.

Palabras clave: enfermedad de Ménière, trastorno depresivo, vértigo, tinnitus, hipoacusia.

\begin{abstract}
Introduction: There is a relationship between Ménière's disease (MD) and psychiatric disorders. Aim: To assess whether MD patients have a higher prevalence of depressive comorbidity compared to that described for the national general population. Material and Method: Cross-sectional analytical study between 2009-2016, for patients seen at the Department of Otolaryngology at the Barros Luco Trudeau Hospital with a definitive MD diagnosis. The functional level scale was applied for MD, tinnitus handicap inventory, Goldberg screening for depression, and an evaluation by a psychiatrist. Results: 64 patients were evaluated. Average age was 52.5 years. The prevalence of depressive disorder was $71.8 \%$. Of these, $71.7 \%$ were diagnosed before the study and the remaining $28.3 \%$ during the study. Conclusion: The prevalence of depressive comorbidity in MD is higher than that described for the national general population. Determine if $M D$ or each symptom separately is the cause of this prevalence requires other studies.
\end{abstract}

Keywords: Ménière's disease, depressive disorder, vertigo, tinnitus, hearing loss.

\author{
${ }^{1}$ Servicio de \\ Otorrinolaringología, Hospital \\ Barros Luco Trudeau. \\ Santiago, Chile. \\ ${ }^{2}$ Facultad de Medicina, \\ Universidad Nacional Andrés \\ Bello. Santiago, Chile. \\ 3ervicio de Psiquiatría de \\ Enlace, Hospital Barros Luco \\ Trudeau. Santiago, Chile. \\ ${ }^{4}$ Facultad de Psicología, \\ Universidad San Sebastián. \\ Santiago, Chile. \\ ${ }^{5}$ Departamento de \\ Otorrinolaringología, Facultad \\ de Medicina, Universidad de \\ Chile. Santiago, Chile.
}

Los autores declaran no tener conflictos de interés.

Recibido el 8 de julio de 2020 . Aceptado el 9 de septiembre de 2020.

\section{Correspondencia:}

Romina Ovalle A.

Gran Avenida 3204, San

Miguel.

Santiago, Chile.

Email: romina.ovalle.alcaino@ gmail.com

\section{Introducción}

La enfermedad de Ménière (EM) es una patología crónica del oído interno de origen idiopático, que se caracteriza por la presencia de crisis de vértigo recurrentes y espontáneas, hipoacusia fluctuante, tinnitus y plenitud ótica ${ }^{1}$. Fue definida en 1972 por el Comité de Equilibrio de la Academia Americana de Otorrinolaringología como una patología que afecta el laberinto membranoso del oído interno, con un sustrato patológico de distensión del sistema endolinfático por hidropesía ${ }^{1}$. Si bien el diagnóstico definitivo se realiza con el 
análisis histopatológico post mortem del hueso temporal, varias sociedades científicas elaboraron un consenso y estipularon en el año 2015 que el diagnóstico "definitivo" se define con los siguientes criterios"

A. Dos o más episodios de vértigo espontáneo con una duración entre 20 minutos y 12 horas.

B. Hipoacusia neurosensorial de frecuencias bajas y medias, documentada con audiometría en un oído, definiendo el oído afectado en al menos una ocasión, antes, durante o después de uno de los episodios de vértigo.

C. Síntomas auditivos fluctuantes (hipoacusia, tinnitus o plenitud) en el oído afectado.

D. No existe otro diagnóstico vestibular que explique mejor los síntomas.

Esta patología puede comenzar a cualquier edad, siendo muy poco frecuente en niños y adolescentes, iniciándose la sintomatología generalmente en la tercera y cuarta década de la vida ${ }^{1}$. La incidencia exacta de la EM es difícil de determinar porque no es de declaración obligatoria y porque es tratada por diversos especialistas (otorrinolaringólogo, neurólogo, médicos de familia), sin embargo, la incidencia podría variar de 10 a 150 por cada 100.000 personas $^{3-5}$. Es una enfermedad cuya evolución varía ampliamente de persona a persona, pudiendo llegar a ser incapacitante en algunos casos con un alto costo socioeconómico, debido al número de licencias médicas otorgadas y jubilaciones por enfermedad. Todos los pacientes, ya sea con tratamiento médico o quirúrgico, presentan alguna alteración emocional en el transcurso de los años debido a la severidad de los síntomas vertiginosos, presencia de tinnitus y/o pérdidas auditivas con una difícil adaptación de audífonos ${ }^{3}$.

En la práctica médica se ha observado que los factores psicológicos desempeñan un papel importante en el curso del vértigo y, en particular, la EM. Existen diversas investigaciones desde el punto de vista psicosomático, pero con limitado nivel de evidencia, dirigidas a determinar la existencia de elementos psicosomáticos en la etiopatogenia de la enfermedad, así como el resultado somato-psíquico que ésta genera. Dentro de los diagnósticos en salud mental más encontrados en la EM están los trastornos ansiosos y depresivos ${ }^{6-11}$.

\section{Objetivo}

El objetivo general de nuestro estudio es evaluar la prevalencia de trastorno depresivo mayor en pacientes chilenos atendidos en el Servicio de Otorrinolaringología del Hospital Barros Luco Trudeau (HBLT), diagnosticados con EM definitivo entre los años 2009-2016.

\section{Material y Método}

Se realizó un estudio analítico transversal entre los años 2009-2016, en pacientes chilenos mayores de 18 años del Servicio de Otorrinolaringología del HBLT con diagnóstico de EM definitivo según los criterios ya descritos ${ }^{2}$. Se excluyeron a pacientes con enfermedades del sistema nervioso central o enfermedad psiquiátrica (excepto trastorno ansioso y/o depresivo). Se calculó el tamaño muestral de 63 pacientes con un nivel de significancia de $5 \%$ y una potencia de $80 \%$. La muestra en este estudio fue de 64 pacientes. Para el análisis estadístico usamos el programa STATA 12.0, con test de Fisher.

El proceso de reclutamiento de participantes para el estudio se realizó a través de la revisión de la base de datos del Departamento de Otoneurología del HBLT, que cuenta con un formato de evaluación consistente en anamnesis, examen físico, examen de VIII par craneal y conclusión médica realizada por otoneuróloga. Se analizaron los resultados de estas evaluaciones que cumplieran con los criterios de inclusión de la muestra, anteriormente mencionados. En aquellos casos que cumplieron con los criterios de ingreso, se les invitó a participar de este estudio mediante una llamada telefónica. Aquellas personas que aceptaron se citaron a control médico con otorrinolaringólogo en donde se les explicó el consentimiento informado y se les aplicaron los instrumentos: Escala del nivel funcional del paciente con enfermedad de Ménière de la AAS-NHS y el test de incapacidad provocada por el tinnitus usando el tinnitus handicap inventory (THI) homologado lingüísticamente para la población chilena en el año $2006^{12}$. En esta instancia, además, se les preguntó si fueron diagnosticados alguna vez en su vida de trastorno depresivo, el año de diagnóstico 
y si permanecían en tratamiento. Los pacientes que respondieron afirmativamente a esta pregunta no continuaron participando de este estudio. Al resto que continuó en el estudio, se les aplicó el test de tamizaje de depresión de Goldberg ${ }^{13}$. Los pacientes que resultaron negativo para el tamizaje de depresión no siguieron participando del estudio, mientras que los que resultaron positivos en este tamizaje fueron citados a evaluación con psiquiatra para confirmar o descartar el diagnóstico mediante una evaluación semiestructurada enfocada a comorbilidad depresiva. En caso de confirmar el trastorno depresivo, se inició el manejo psiquiátrico respectivo y se derivó el paciente a su centro de salud para ser ingresado al sistema de Garantías Explícitas en Salud (GES) ${ }^{13}$.

Las variables tabuladas en este estudio fueron: edad, sexo, comorbilidades, diagnóstico de depresión previo al estudio, y si fue antes o después de iniciada la EM, año de inicio de sintomatología EM, año de diagnóstico EM, años de evolución EM, número de crisis en últimos 6 meses, hallazgos en octavo par, tratamiento de la $\mathrm{EM}$, resultados de la última audiometría posdiagnóstico, resultados de la escala del nivel funcional del paciente con EM de la AAS-NHS y del THI, características del tinnitus, resultado en test de tamizaje de trastorno depresivo de Goldberg, resultado evaluación psiquiátrica, conducta del psiquiatra con los pacientes diagnosticados (activación sistema GES, derivación a atención primaria). Este estudio contó con la aprobación del comité de ética del Servicio de Salud Metropolitano Sur, Santiago, Chile.

\section{Resultados}

De un total de 72 pacientes con diagnóstico de EM entre los años 2009 y 2016, se evaluó a 64 pacientes, de los cuales un $56,2 \%$ correspondieron al sexo femenino. La edad promedio al momento del diagnóstico de EM fue de 53 años con un rango de edad de 41 a 60 años. El $75 \%$ presentaba comorbilidades como hipotiroidismo, diabetes mellitus, hipertensión, dislipidemia, tabaquismo y artrosis. El tiempo promedio de evolución desde el inicio de los síntomas de EM hasta la intervención fue de 4,7 años (rango: 1-7 años). El 43,8\% refirió tener crisis de vértigo en los 6 meses previo al estudio y todos indicaron utilizar como tratamiento difenidol o cinarizina en caso de crisis. Solo un $37,5 \%$ se encontraba utilizando betahistina de forma permanente como tratamiento de base.

El $100 \%$ de los pacientes presentaba tinnitus, descrito como unilateral, persistente y de tonalidad grave, un $28 \%$ refirió espontáneamente que durante las crisis vertiginosas o frente a situaciones de estrés el tinnitus cambiaba a tonalidad aguda. En la prueba calórica la alteración más frecuente fue la presencia de una paresia vestibular unilateral y en la audiometría una hipoacusia sensorioneural unilateral ascendente (Tablas 1 y 2) con un promedio tonal puro (PTP) promedio en oído derecho de $22,6 \mathrm{~dB}$ y en oído izquierdo de $41,6 \mathrm{~dB}$.

El 51,5\% de los participantes (12 mujeres y 21 hombres) fue diagnosticado con trastorno depresivo mayor antes del estudio o dentro del primer año de evolución de la EM. A los 31 pacientes restantes se les aplicó el test de tamizaje para depresión, para detectar casos probables que, posteriormente, serían evaluados por psiquiatra para confirmar diagnóstico. De éstos, 18 (6 mujeres y 1 hombre) presentaron un puntaje indicativo de probable trastorno depresivo. Los 13 pacientes restantes (7 mu-

Tabla 1. Hallazgos encontrados en la prueba calórica

\begin{tabular}{lc}
\hline Normal & $15,6 \%(10 / 64)$ \\
\hline Paresia vestibular unilateral & $60,9 \%(39 / 64)$ \\
Lesión combinada unilateral & $23,5 \%(15 / 64)$ \\
Dirección preponderante & $0 \%$
\end{tabular}

Tabla 2. Hallazgos encontrados en la última audiometría realizada

Audiometría tonal

\begin{tabular}{lll} 
HSN unilateral & Ascendente & $71,4 \%(35 / 49)$ \\
$76,6 \%(49 / 64)$ & Descendente & $28,6 \%(14 / 49)$ \\
& Plana & $0 \%$ \\
HSN bilateral asimétrica & Ascendente & $0 \%$ \\
$23,4 \%(15 / 64)$ & Descendente & $73,3 \%(11 / 15)$ \\
& Plana & $26,7 \%(4 / 15$ \\
\hline HSN: hipoacusia sensorio-neural. &
\end{tabular}




\begin{tabular}{|c|c|c|c|}
\hline Grado & & $\begin{array}{l}\text { Depresivos } \\
(n=46)\end{array}$ & $\begin{array}{l}\text { No depresivos } \\
\quad(n=18)\end{array}$ \\
\hline 1 = Muy leve & $\begin{array}{l}\text { Tinnitus percibido solo en ambiente silencioso, fácilmente } \\
\text { enmascarable, casi nunca perturba al paciente }\end{array}$ & $4,3 \%(2)$ & $27,8 \%(5)$ \\
\hline 2 = Leve & $\begin{array}{l}\text { Tinnitus enmascarable por el ruido ambiente y olvidado durante } \\
\text { la actividad diaria }\end{array}$ & $37 \%(17)$ & $55,6 \%(10)$ \\
\hline $3=$ Moderado & $\begin{array}{l}\text { Tinnitus percibido a pesar del ruido ambiente, pero no dificulta } \\
\text { AVD. Sin embargo, molesta en reposo y puede dificultar la } \\
\text { conciliación del sueño }\end{array}$ & $17,4 \%(8)$ & $11,1 \%(2)$ \\
\hline 4 = Severo & $\begin{array}{l}\text { Tinnitus siempre percibido, interfiere en AVD, dificulta el reposo } \\
\text { y el sueño. Consultas frecuentes a especialistas }\end{array}$ & $34,7 \%(16)$ & $5,5 \%(1)$ \\
\hline 5 = Catastrófico & $\begin{array}{l}\text { Afección peor que grado 4, principalmente el insomnio, es } \\
\text { posible encontrar patologías psiquiátricas asociadas }\end{array}$ & $6,6 \%(3)$ & 0 \\
\hline
\end{tabular}

jeres y 6 hombres) completaron el estudio en este punto, ya que no presentaron sospecha de trastorno depresivo en el tamizaje aplicado.

La evaluación psiquiátrica confirmó el diagnóstico de trastorno depresivo mayor en 13 (12 mujeres y 1 hombre) de los 18 pacientes con probable trastorno depresivo. En todos los casos confirmados, el psiquiatra inició un tratamiento farmacológico según correspondiera y realizó la notificación GES, informe de proceso diagnóstico (IPD) y derivación a atención primaria de salud. Se encontró trastorno depresivo mayor en 46 de los 64 pacientes con EM. La prevalencia calculada de trastorno depresivo mayor fue de un 71,9\%. Al comparar este resultado con la prevalencia en la población general chilena, que corresponde a un $9 \%{ }^{14}$, resulta más alta.

Se encontró que el 78,5\% de los hombres y el $66,7 \%$ de las mujeres presentaron trastorno depresivo, sin existir una diferencia estadísticamente significativa entre ambos grupos (test de Fisher $p=0,221$ ). Al evaluar la incapacidad provocada por el tinnitus con el THI (Tabla 3 ) el 10,9\% (7/64) presenta un tinnitus muy leve o grado 1 , el $42,1 \%(27 / 64)$ un tinnitus leve o grado 2 , el $15,6 \%(10 / 64)$ un tinnitus moderado o grado 3 , el $26,5 \%$ (17/64) un tinnitus severo o grado 4 y el $4,9 \%$ (3/64) un tinnitus catastrófico o grado 5 , siendo mayor la incapacidad en el grupo con depresión comparado con los no depresivos (test de Fisher $p<0,001$ ).

$\mathrm{Al}$ evaluar subjetivamente el impacto de la EM en la vida diaria a través de la encuesta del nivel de funcionalidad de la EM (Tabla 4), todos los pacientes refirieron que el vértigo los afecta en actividades de su vida diaria, no existiendo diferencia significativa entre los grupos con trastorno depresivo y sin depresión (test de Fisher $p=0,197$ ).

\section{Discusión}

Al igual que lo descrito en la literatura, la población estudiada con EM corresponde en su mayoría a mujeres entre 40 y 60 años. El $56,2 \%$ de los pacientes refirió no haber tenido crisis de vértigo en los últimos 6 meses, presentándose en ellos solo tinnitus y la hipoacusia intermitente o progresiva. El 43,8\% restante sigue presentando la triada clásica de la EM (vértigo, hipoacusia y tinnitus). Esta persistencia del vértigo se puede explicar por la historia natural de la enfermedad con una evolución de menos de 4 años y por no tener acceso al tratamiento con betahistina en forma regular debido a su alto costo ${ }^{1}$.

En la población chilena los cuadros depresivos se dan con mayor frecuencia en las mujeres, sin embargo, en los pacientes con EM se encontró mayor número de hombres que 


\begin{tabular}{|c|c|c|c|}
\hline Nivel & & $\begin{array}{l}\text { Depresivos } \\
(n=46)\end{array}$ & $\begin{array}{l}\text { No depresivos } \\
\quad(n=18)\end{array}$ \\
\hline Nivel 1 & El vértigo no afecta en nada mi vida & $0 \%$ & $0 \%$ \\
\hline Nivel 2 & $\begin{array}{l}\text { Cuando tengo vértigo debo cesar mi actividad en ese momento, luego } \\
\text { continúo normalmente y no afecta a mis planes }\end{array}$ & $23,9 \%(11)$ & $38,9 \%(7)$ \\
\hline Nivel 3 & Ídem a nivel 2, pero me hace cambiar algunos planes & $13 \%(6)$ & $27,7 \%(5)$ \\
\hline Nivel 4 & $\begin{array}{l}\text { Puedo hacer una vida normal, pero me obliga a un gran esfuerzo, } \\
\text { constantemente he de ajustar mis actividades }\end{array}$ & $26,1 \%(12)$ & $5,6 \%(1)$ \\
\hline Nivel 5 & $\begin{array}{l}\text { No puedo trabajar, ni conducir ni mantener a mi familia. Soy incapaz de } \\
\text { realizar la mayoría de las actividades diarias. Estoy incapacitado }\end{array}$ & $30,4 \%(14)$ & $22,2 \%(4)$ \\
\hline Nivel 6 & $\begin{array}{l}\text { Estoy de baja laboral durante al menos } 1 \text { año o recibo una compensación } \\
\text { económica por ello }\end{array}$ & $6,6 \%(3)$ & $5,6 \%(1)$ \\
\hline
\end{tabular}

de mujeres con trastorno depresivo por lo que pueden haber otros factores involucrados en la etiopatogenia del cuadro depresivo ${ }^{13,14}$. En nuestro estudio encontramos una alta prevalencia de trastorno depresivo en pacientes con EM, sugiriendo alguna relación ${ }^{3-5,13,14}$. Existiría un círculo vicioso entre la interacción de los síntomas orgánicos somáticos de la EM y el estrés resultante por las crisis vertiginosas. Las crisis de vértigo pudiesen constituir un factor precipitante de estados de estrés subjetivos empeorando el estado emocional en los pacientes. La ansiedad resultante provocaría síntomas ansioso-depresivos, probablemente a través de una disfunción del sistema nervioso autónomo ocasionado por el aumento de los niveles de hormonas relacionadas con el estrés ${ }^{6}$. De hecho, algunos estudios retrospectivos y serie de casos han evaluado diversas metodologías de la psicoterapia (técnicas cognitivo-conductuales, tratamiento autógeno, terapia grupal) en pacientes con EM, todos ellos con resultados favorables ${ }^{6-11}$.

Por otra parte, el vértigo recurrente puede llegar a ser un síntoma altamente incapacitante que pudiese explicar por sí mismo un trastorno depresivo sin necesidad de formar parte de la EM. Sin embargo, llama la atención que mientras un $43,8 \%$ de los pacientes refieren persistir con vértigo al momento de la evaluación, el $71,9 \%$ de los pacientes presentan un trastorno depresivo. Esta diferencia permite interpretar que no solo el vértigo puede generar malestar psíquico en estos pacientes, sino que también los otros síntomas de la EM, principalmente el tinnitus presente en el $100 \%$ de los pacientes, de los cuales el $47 \%$ refiere tener un tinnitus desde grado moderado a catastrófico. Determinar si la EM es la causante de esta alta prevalencia de trastornos depresivos, o si éstos son causados por cada uno de sus síntomas por separado, requerirá de nuevos estudios.

\section{Conclusión}

La prevalencia de comorbilidad depresiva en la EM es alta. El tinnitus está presente en todos los pacientes de la muestra. No todos los pacientes logran estar compensados de sus crisis de vértigo en los últimos 6 meses a pesar del tratamiento de la EM. Se sugiere considerar una evaluación psiquiátrica en algún momento de la EM por la alta prevalencia de depresión encontrada.

\section{Bibliografía}

\footnotetext{
1. Saenz M. Enfermedad de Ménière. Rev

Otorrinolaringol Cir Cabeza Cuello. 2002;62:289-298.

2. López-Escamez JA, Carey J, Chung WH, et al.
} 
Criterios diagnósticos de enfermedad de Menière. Documento de consenso de la Bárány Society, la Japan Society for Equilibrium Research, la European Academy of Otology and Neurotology (EAONO), la American Academy of Otolaryngology-Head and Neck Surgery (AAO-HNS) y la Korean Balance Society. Acta Otorrinolaringol Esp. 2016;67(1):1-7. doi: 10.1016/j. otorri.2015.05.005.

3. Monsell EM, Balkany TA, Gates GA. Committee on hearing and equilibrium guidelines for the diagnosis and evaluation of therapy in Ménière's disease. Otalaryngol Head Neck Surg. 1995;113:181-185.

4. Dinces E, Deschler D, Park L. Meniere Disease. UpToDate. 2013.

5. Goto F, Nakai K, Ogawa K. Application of autogenic training in patients with Ménière disease. Eur Arch Otorhinolaryngol. 2011;268(10):1431-1435.

6. Naber CM, Water-Schmeder O, Bohrer PS, Matonak $\mathrm{K}$, Bernstein AL, Merchant MA. Interdisciplinary treatment for vestibular dysfunction: the effectiveness of mindfulness, cognitive-behavioral techniques, and vestibular rehabilitation. Otolaryngol Head Neck Surg. 2011; 145(1):117-124.

7. Savastano M, Marioni G, Aita M. Psychological characteristics of patients with Ménière's disease compared with patients with vertigo, tinnitus, or hearing loss. Ear Nose Throat J. 2007;86(3):148-156.

8. Yardley L, Kirby S. Evaluation of booklet-based self-management of symptoms in Ménière disease: a randomized controlled trial. Psychosom Med. 2006; 68(5):762-769.

9. Kirby SE, Yardley L. Cognitions associated with anxiety in Ménière's disease. J Psychosom Res. 2009; 66(2):111-118

10. Coelho DH, Lalwani AK. Medical management of Ménière's disease. Laryngoscope. 2008;118(6):10991108. doi: 10.1097/MLG.0b013e31816927f0.

11. Kirby SE, Yardley L. Understanding psychological distress in Meniere's disease: a systematic review. Psychol Health Med. 2008; 13(3):257-273.

12. Peña Martínez A. Evaluation of discapacity generated by tinnitus: National linguistic homologation of Tinnitus Handicap Inventory (THI). Rev Otorrinolaringol Cir Cabeza Cuello. 2006;66:232-235.

13. Guía Clínica Garantías Explícitas en Salud: Depresión en mayores de 15 años y más. Serie guías clínicas MINSAL. Chile, 2013.

14. Vicente B, Rioseco P, Saldivia S, Kohn R, Torres S. Estudio chileno de prevalencia de patología psiquiátrica (DSM-III-R/CIDI) (ECPP). Rev Med Chile. 2002; 130(5):527-536. 